\title{
Optimal Design of D-type Plastic Fibers for best sensitivity of SPR Sensors
}

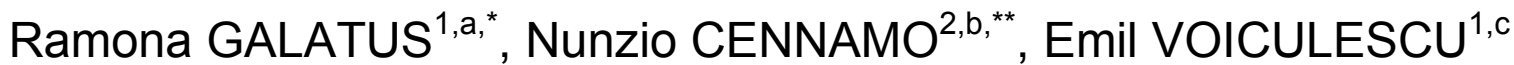 \\ ${ }^{1}$ Basis of Electronics Department, Optoelectronic and Optical Integrated Components Group, \\ Technical University of Cluj-Napoca, 400114, Romania; \\ ${ }^{2}$ Department of Industrial and Information Engineering, Second University of Naples, Via Roma 29, \\ 81031 Aversa, Italy; \\ åRamona.Galatus@bel.utcluj.ro, ㅁnunzio.cennamo@unina2.it, ㅌEmil.Voiculescu@bel.utcluj.ro,
}

Keywords: D-type plastic optical fiber, SPR sensor, numerical simulation for the optimal sensitivity, multi-layered, experimental results for light intensity measurements, high-precision sensor, Lorenz-Drude model.

\begin{abstract}
A design method for a SPR (surface plasmon resonance) sensor based on a D-type plastic optical fiber (POF) geometry in a configuration of a multi-layered scheme is proposed in this paper. The numerical simulation for the optimal sensitivity with spectral interogation was performed in order to choose the practical implementation geometry of the SPR sensor. A side-polish to the half of the fiber's PMMA core, with about $10 \mathrm{~mm}$ in length is made and a Microposit 1813 buffer and gold layer deposition is used for sample fabrication. The effect of different thickness of the layers in different multi-layered configuration has been investigated. The proposed setup for sensor test, measures the light intensity instead of phase difference. It can be used for refractive index changes (1.33-1.40) in real time detection of the substances for different biosensing applications. The proposed sensor is simple to use, has small size, it works on small analyt-sample size and it is cost effective for specific non-invasive application users.
\end{abstract}

\section{Introduction}

The free-label detection of chemical analyt medium using light-excited surface plasmon resonance (SPR) is nowadays a broadly used technique. It is further an object of the invention to provide a solution for a SPR-based device that is easy to manufacture, cost-effective and simple to use for the laboratory specialists. A low-cost plastic optical fiber (POF) solution was considered [1] instead of classically most widely used Kretschmann prism-coupling geometry. The POF fiberbased SPR has many advantages over prism-based model such as flexible optical design because of the easy modeled plastic materials, development of remote sensing setup, it may reduce the cost and dimensions of the device and there exists the possibility of integration with optoelectronic devices (eventually leading to "lab on a chip"), to be used in continuous analysis in real-time (not just one sample at a time) and in situ monitoring.

In this paper a SPR sensor response with different stack-layered geometry has been theoretically investigated, in order to obtain an optimal geometry with a good sensitivity and resolution ratio. The design method is used in order to select the optimal manufacturing parameters for the sensor. The SPR biosensor was developed and tested at the Department of Information Engineering, Second University of Naples, Italy and the results was published in [1]. It was used in this study for performance comparison between theoretical and experimental results; the results are in close concordance. The numerical results are obtained based on transfer matrix formalism using Matlab simulations. The preferred mode of surface plasmon wave (and hence the marker) detection is based on spectral analysis of insertion loss experienced in the fiber device. SPW (surface plasmon wave) results in a peak of insertion loss at certain wavelength (identifying type of the marker) and amplitude (indicating its concentration). Suitable interrogator is able to detect increase of insertion loss at certain wavelength corresponding to SPW resonance against reference at other wavelength(s) not influenced by the SPW. 
The POF has a PMMA core of $980 \mu \mathrm{m}$ and a fluorinated polymer cladding of $20 \mu \mathrm{m}$. The D-type geometry enabling it to be a candidate for successful biosensors implementation; the planar gold layer that permits easy-binding process of antibody.

\section{Theory}

The propagation of the free electron density oscillation on the surface of a dielectric medium, subjected to an incident light, in contact with a metal gives rise to a surface plasmon wave (SPW). The SPW can only be supported along the boundary of two materials layers with opposite signs of the real parts of the dielectric constants, such as a dielectric and a metal. Due to the intrinsic electron oscillation damping loss in the metal the SPW attenuate rapidly during the layered-path propagation. In a metal electron oscillates with plasma frequency, $\omega_{\mathrm{p}}=4 \pi \mathrm{ne}^{2} / \mathrm{m}$ [2] (where $\mathrm{n}$ is the electron number density, e is the electron charge and $\mathrm{m}$ is the electron mass). The resonance condition for the excitation of surface plasmon wave (Eq.1) is obtained considering the dispersion relation for surface waves. The output signal demonstrates a dip at a particular wavelength known as resonance wavelength. The propagation constant of the evanescent wave generated as a result of Attenuated Total Reflection (ATR) of the light incident at an angle $\theta_{\text {incident }}$ through a light coupling device (in this case optical fiber) of refractive index $\mathrm{n}_{\text {core }}$, is equal to that of the SPW:

$$
K_{\mathrm{ATR}}=\text { Kplasmon. }
$$

where

$$
\begin{aligned}
& K_{\mathrm{ATR}}=K_{0} n_{\text {core }} \sin \theta_{\text {incident }} \\
& K_{\text {plasmon }}=\operatorname{Re}\left[K_{0}\left(\frac{\varepsilon_{\text {metal }} n_{\text {sen } \sin g}^{2}}{\varepsilon_{\text {metal }}+n_{\text {sen } \sin g}^{2}}\right)^{1 / 2}\right] ; \\
& K_{0}=\frac{2 \pi}{\lambda}
\end{aligned}
$$

with the following notation: $\varepsilon_{\text {metal }}$-real part of the metal dielectric constant, $n_{\operatorname{sen} \sin g}$ - refractive index of the sensing layer.

The ultra-thin metallic layers $(20 \mathrm{~nm}<d<60 \mathrm{~nm})$ is stacked alternatively with positive photoresist (Microposit S1800).

The complex value of dielectric constant was calculated according with Lorenz-Drude model (LDm) [6]. The LDm is often used for parameterization of the optical constants of metals because provides a dielectric function with good correlation with the experimentally measured dielectric functions of metals over a wide frequency range.

$$
\varepsilon(\omega)=\varepsilon_{r, \infty}+\sum_{k=0}^{K} \frac{f_{k} \omega_{p}^{2}}{\omega_{k}^{2}-\omega^{2}+j \omega \Gamma_{k}}
$$

where:

- $\varepsilon_{r, \infty}$ is the dielectric constant at infinite frequencies (free-electron or Drude model),

- $\omega_{p}$ is the plasma frequency already defined above, and 
- $K$ is the number of oscillators with resonance frequency $\omega_{k}$, strength $f_{k}$ and lifetime $1 / \Gamma_{k}$. It is used the values from [7].

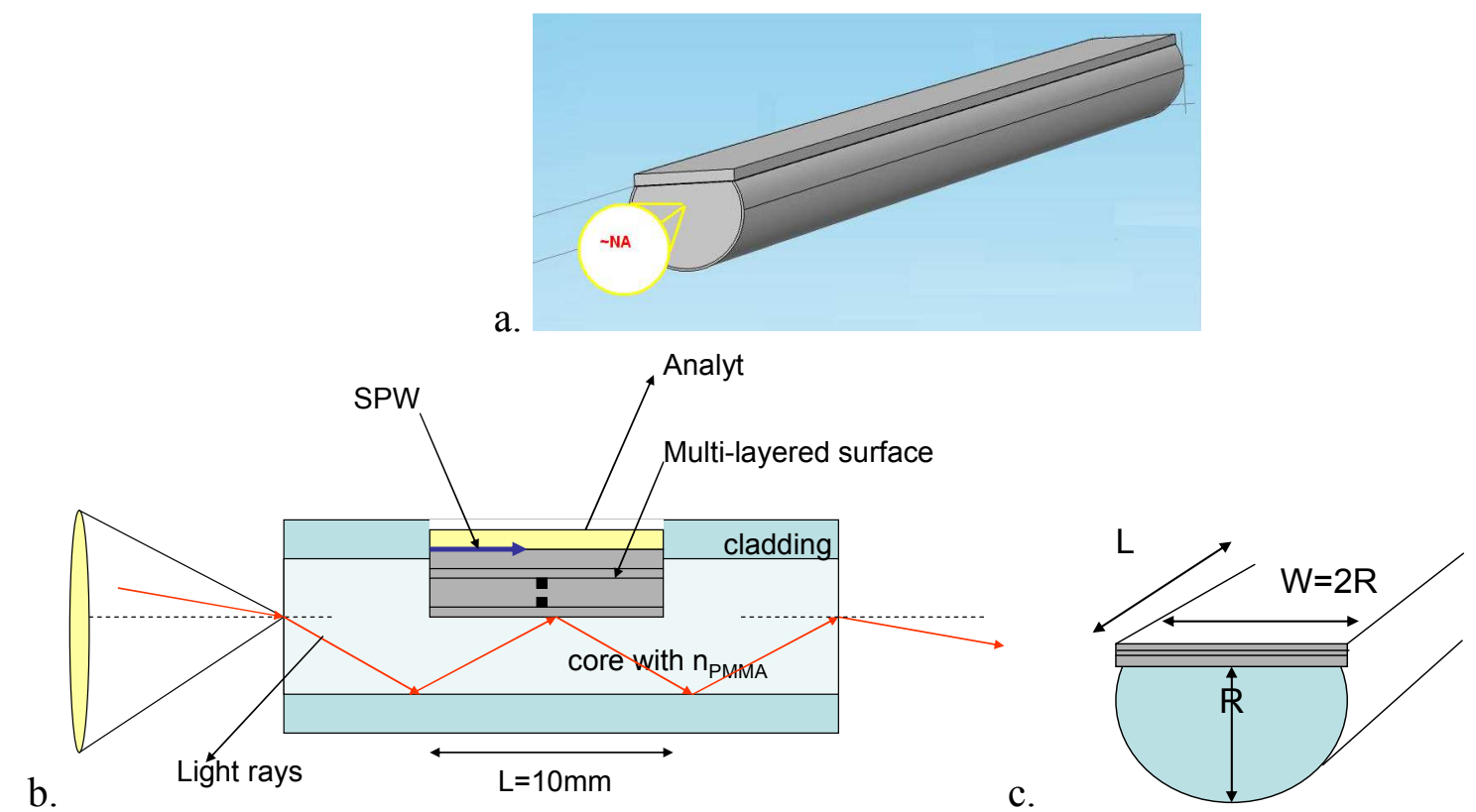

Fig 1. D-type SPR sensing region, $\mathrm{L}=10 \mathrm{~mm}$. a. 3D model, b. 2D longitudinal section, c. $2 \mathrm{D}$ transversal section

According with [3], the dispersion for PMMA material, for fiber core is:

$\mathrm{n}^{2}(\lambda)=2.399964-8.308636 \times 10^{-2} \lambda^{2}-1.919569 \times 10^{-1} \lambda^{-2}+8.720608 \times 10^{-2} \lambda^{-4}-1.666411 \times 10^{-2} \lambda^{-6}+1.1$ $69519 \times 10^{-3} \lambda^{-8}$

For Microposit S1800 photoresist, the dispersion curve is mentioned in [4].

The fiber has locally removed cladding along half circumference. POF is composed of soft materials and a simple procedure of side-polishing with polishing papers ( $8 \mu \mathrm{m}$ to $1 \mu \mathrm{m})$ was adopted since the dimensions of samples can be easily controlled. Ordinary fibers are no longer cylindrically symmetrical after side-polished, called also D-type fiber [9]. The state of linearly polarized light oriented parallel to a polished surface is the TE mode. Its orthogonal state is the TM mode that has contribution to plasmonic effect ( $p$-wave).

Consider the N-layer model as shown in Fig 1.b, and Fig 2 in detailed, with the layers stacked along the y-axis, the transfer matrix formalism for a multilayer system is applied [5].

The reflection coefficient (reflectance) of the $p$-polarized light is given by:

$$
R_{p}=\left|r_{p}\right|^{2}=\left|\frac{\left(M_{11}^{p}+M_{12}^{p} \xi_{N}^{p}\right) \xi_{o}^{p}-\left(M_{21}^{p}+M_{22}^{p} \xi_{N}^{p}\right) \xi_{N}^{p}}{\left(M_{11}^{p}+M_{12}^{p} \xi_{N}^{p}\right) \xi_{o}^{p}+\left(M_{21}^{p}+M_{22}^{p} \xi_{N}^{p}\right) \xi_{N}^{p}}\right|^{2}
$$

and for the $s$-polarized light is given by:

$$
R_{s}=\left|r_{s}\right|^{2}=\left|\frac{\left(M_{11}^{s}+M_{12}^{s} \xi_{N}^{s}\right) \xi_{o}^{s}-\left(M_{21}^{s}+M_{22}^{s} \xi_{N}^{s}\right) \xi_{N}^{s}}{\left(M_{11}^{s}+M_{12}^{s} \xi_{N}^{s}\right) \xi_{o}^{s}+\left(M_{21}^{s}+M_{22}^{s} \xi_{N}^{s}\right) \xi_{N}^{s}}\right|^{2}
$$

where $[\mathrm{M}]$ is the characteristic matrix of the layered system: 


$$
[M]=\left[\begin{array}{ll}
M_{1,1} & M_{1,2} \\
M_{2,1} & M_{2,2}
\end{array}\right]=\prod_{k=1}^{N-1}\left(\left[\begin{array}{cc}
\cos \varsigma_{k} & \frac{-i \sin \varsigma_{k}}{\xi_{k}} \\
-i \xi_{k} \sin \varsigma_{k} & \cos \varsigma_{k}
\end{array}\right]\right)
$$

with:

$-\xi_{k}^{s, p}$ is the optical admittance defined as a function of polarization state and $-\varsigma_{k}$, is the phase factor of the $k$-layer.

$$
\begin{aligned}
& \varsigma_{k}=K_{0} n_{k} \cos \theta_{k}\left(y_{k}-y_{k-1}\right)=K_{0} d_{k} \sqrt{\left(\varepsilon_{k}-n_{\text {core }}^{2} \sin ^{2} \theta_{\text {incident }}\right)} \\
& \xi_{k}^{(s, p)}=\frac{\sqrt{\left(\varepsilon_{k}-n_{\text {core }}^{2} \sin ^{2} \theta_{\text {incident }}\right)}}{\varepsilon_{k}^{s}{ }_{k}}
\end{aligned}
$$

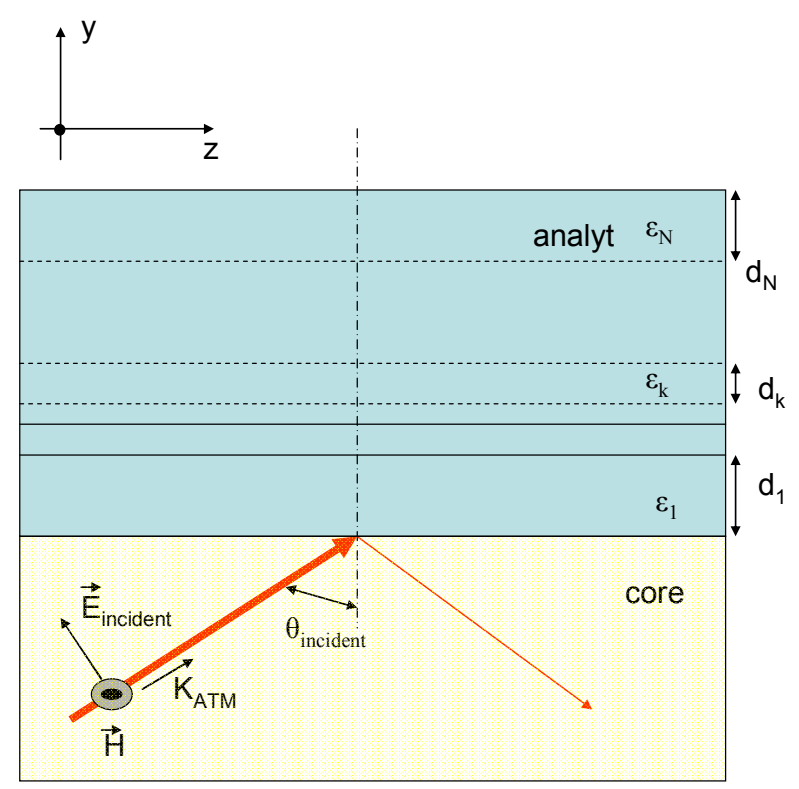

Fig 2. Vectors for $p$-wave (TM-transversal magnetic) at the incident surface of multilayered system

In a general transmission function the transmittance form depends on fiber-optic properties, launching conditions and ray direction. The power at the fiber's output at a specific wavelength can be approximated with integrating the product of the whole light reflectance by the angular power distribution corresponding to the light source used (Eq 10). The distribution is assumed to be uniform simulating along fiber length and TE and TM polarization component equally distributed (skew ray does not have a definite polarization), and both $r_{s}, r_{p}$ was considered:

$$
P_{\text {out }}\left(\lambda, n_{\text {sensing }}\right)=\frac{1}{2}\left(\int_{\theta_{\text {critical }}}^{90^{\circ}} R_{p}^{N} P_{0}\left(\lambda, \theta, n_{\text {sen } \sin g}\right) d \theta+\int_{\theta_{\text {critical }}}^{90^{0}} R_{s}^{N} P_{0}\left(\lambda, \theta, n_{\text {sen } \sin g}\right) d \theta\right)
$$




$$
N=\frac{L W}{R \tan (\theta}
$$

where $\mathrm{N}$ is the number of reflections within the sensitive area, that is calculated as a function of $\mathrm{L}$, length of the sensitive area, $\mathrm{W}$, width of the sensitive area and $\mathrm{D}$, diameter of the fiber, while $r_{s}, r_{p}$ are the reflection coefficient for polarization TE and TM, respectively.

The power distribution arriving at the end-face of the fiber can be expressed as in Eq 12.

$$
P_{0} d \theta \alpha \frac{n_{c o r e}^{2} \sin \theta \cos \theta}{\left(1-n_{\text {core }}^{2} \cos ^{2} \theta\right)^{2}} d \theta
$$

The considered model has a good real data results even it does not take into account scattering from possible roughness of the layer, diffraction or dispersion and mode coupling phenomena because of the small sensing fiber path.

In a light intensity absorption-based fiber optic SPR sensor, normalized transmitted power $(\mathrm{T}(\lambda))$ is determined relatively to the transmitted power obtained for the blank medium $\left(\mathrm{P}_{\text {ref }}(\lambda)\right)$.

$$
T(\lambda)=\frac{P_{o u t}(\lambda)}{P_{r e f}(\lambda)}
$$

The sensor sensitivity can be expressed by calculating the ratio between consecutive shift in resonance wavelength related to unit change in refractive index. The theoretical values are discussed for different multi-layered geometry in order to manufacture de sensor.

$$
S\left(n_{\text {sen } \sin g}\right)=\frac{\delta \lambda_{\text {resonance }}}{\delta n_{\text {sen } \sin g}}\left[\frac{n m}{R I U}\right]
$$

which can be approximated with the variation of minimum power (that correspond to resonance wavelength) related to unit change in refractive index.

The resolution or signal-to-noise ratio (SNR) of the SPR sensor is inversely proportional to the full width at half maximum (the narrower the width the higher the detection accuracy) of the SPR response curve.

$$
\operatorname{SNR}\left(n_{\text {sen } \sin g}\right)=\left[\frac{\delta \lambda_{\text {resonance }}}{\delta \lambda_{F W H M}}\right]_{n_{\text {sen } \sin g}}
$$

\section{Results and discussion}

With the best of our knowledge, a close approach analysis is present in the literature with reference to a silica optical fiber [8] [10] round-type or a sensor based SPR [9] in D-type monomode silica optical fiber with other buffer layer $\left(\mathrm{SiO}_{2}\right)$ between the fiber core and the gold film.

The SPR based on plastic optical fiber (POF) fiber-based has many advantages over silica optical fiber, such as:

- flexible optical design, as easily processed plastic materials are used

- the most common POF material is poly-methyl metacrylate (PMMA), long known for being non-toxic and showing good biological compatibility. 
- PMMA-based POFs are suitable low-cost alternative for light transmission in the visible and near infrared range (approx. 450-850 $\mathrm{nm}$ ) with attenuation below $0.2 \mathrm{~dB} / \mathrm{m}$.

The buffer layer permits to increase the adhesive force between metallic layer and fiber core as a substrate.

Considering the ultra-thin metallic layers deposition $\left(d_{\text {metallic layer }}=20,40,60 \mathrm{~nm}\right)$ embedded in a sensing region within a layered-stack deposition on the plastic core, and analyt refractive index $\mathrm{n}_{\text {sensing }}=\left[\begin{array}{llllllllll}1.332 & 1.34 & 1.35 & 1.36 & 1.37 & 1.38 & 1.39 & 1.40 & 1.41 & 1.418\end{array}\right]$ (corresponding to the data 2-data 11 series in figure legend, with data 1 as etalon), the results depicted in Fig 3-5 was obtained, corresponding to the geometry description in Table 1.

To obtain maximum values of S (sensitivity) and SNR(signal-to-noise ratio), it needs to identify appropriate values of metal film thickness and layer order. The sensing length that is considered has $\mathrm{L}=10 \mathrm{~mm}$. The most used metals for SPR, gold and silver, was considered. A new approach with aluminum deposition has been proposed in [11] and it will consider in a future work.

Because the sensitivity of this device can reach about $0.8-1.2 \times 10^{3} \mathrm{~nm} / \mathrm{RIU}$ for $980 \mu \mathrm{m}$ POF and resolution about 0.08-0.6, it can be used for the refractive index range in interval range 1.33-1.40.

At the present work, the geometry with core/buffer- $\mathrm{d}_{1} /$ gold $-\mathrm{d}_{2} /$ analyt $\left(\mathrm{d}_{2}=40, \mathrm{~d}_{1}=1.5 \mu \mathrm{m}\right)$ was selected for good sensitivity to resolution ratio and experimentally analyzed. Among the 12-th geometry types, the 5-th, 6-th and 12-th reflect good performance parameters, including real-value of the parameters that can be obtained with deposition devices (spin coating and sputtering steps).

In the following graphics there are some selections of the representative results.

To demonstrate the practicability of the proposed method, a laboratory SPR biosensor [1] developed at the Department of Information Engineering, Second University of Naples, Italy was used in this study to compare the theoretical and experimental results. For optical fiber a model no. "PMMA POF D.980/1000, manufactured by Luceat Spa, Torbole Casaglia (BS), Italy was used. The results are in good concordance with the theoretical simulations results (Fig 3 from [1]). For the future work, at the next step, the 6-th and 12-th (the shaded) geometry from the Table 1 will be study for performance comparison

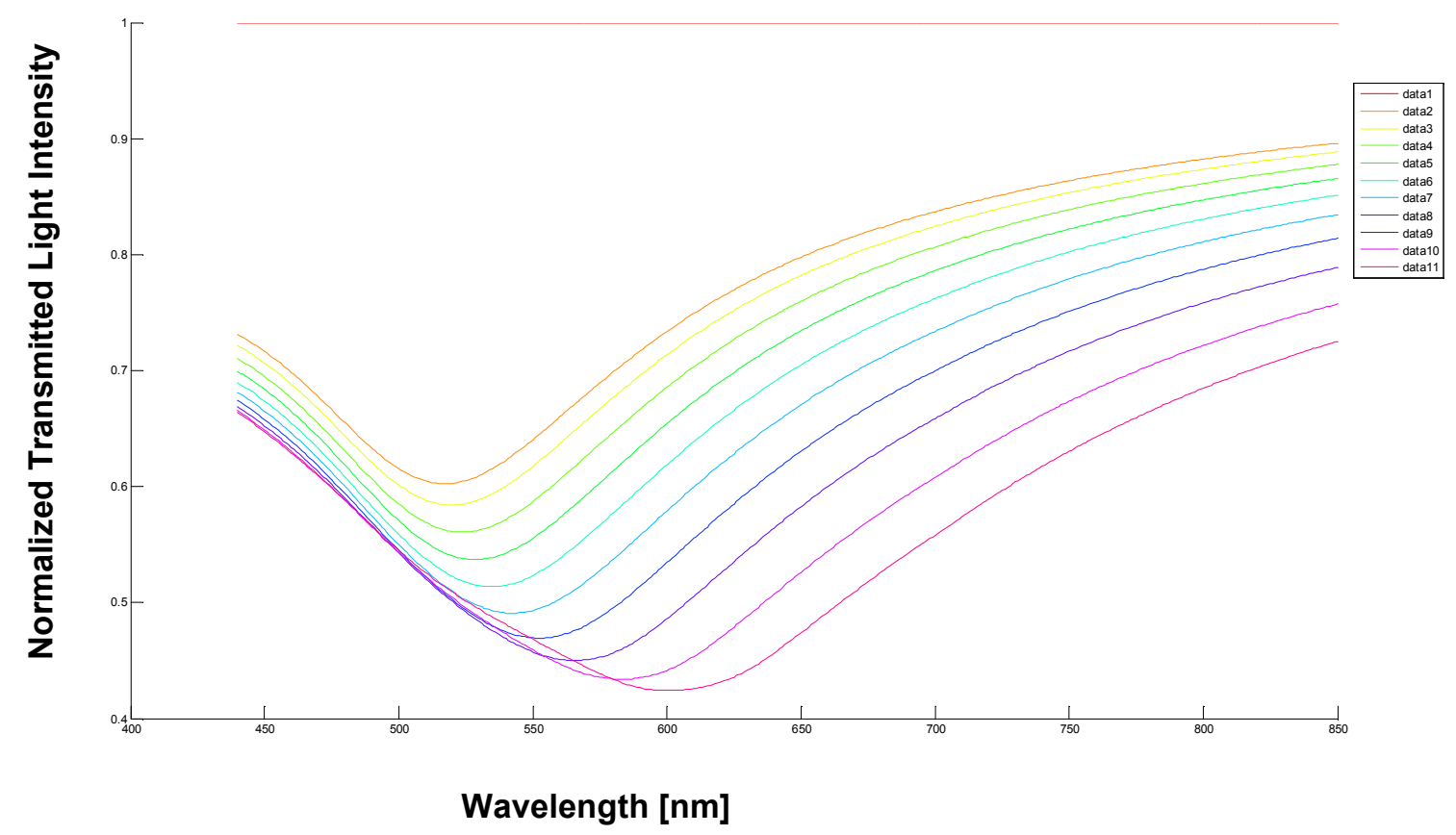

a. spectral response in wavelengths range of interest with different refractive index values (legend) 


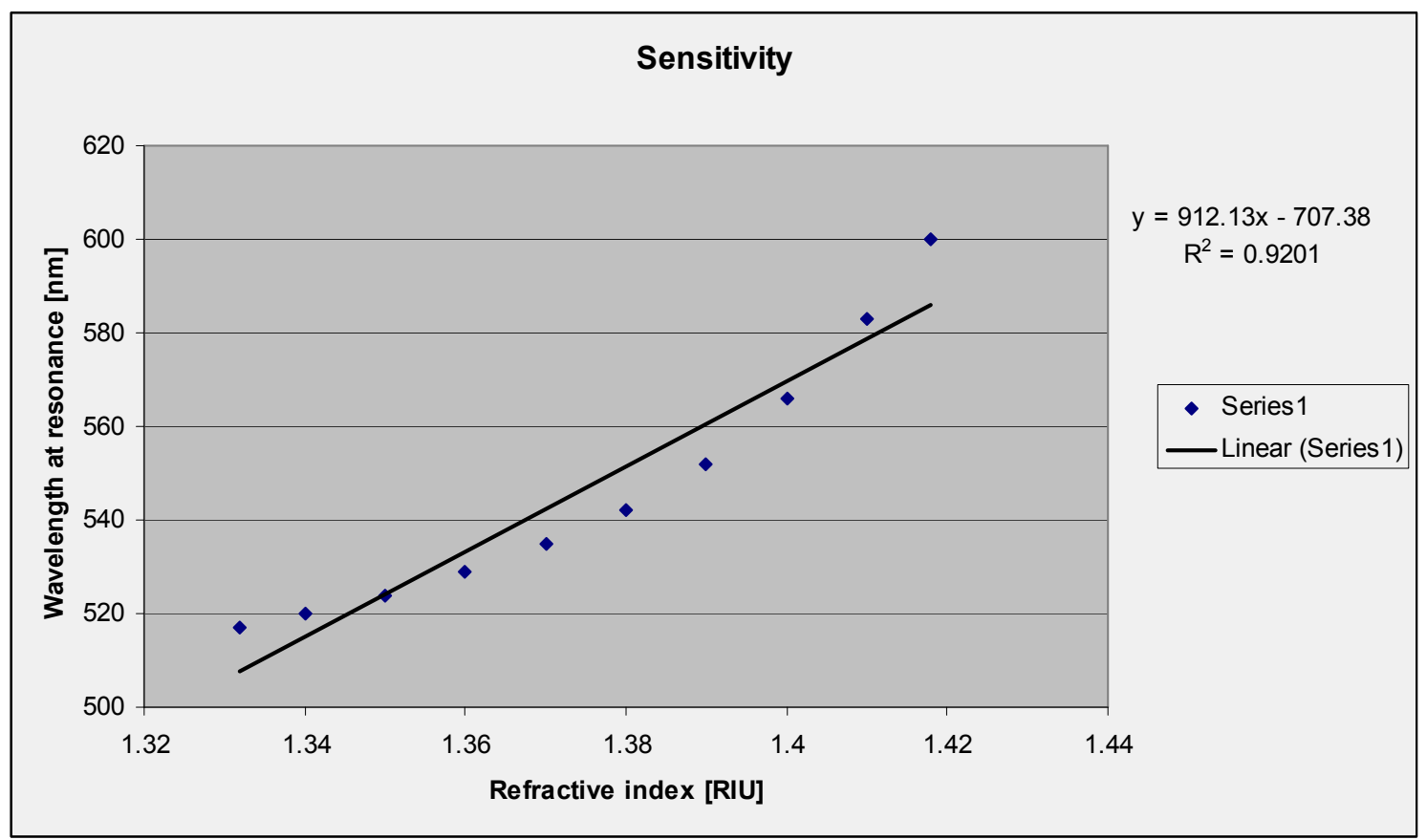

b. Linearity of the SPR response

Fig 3. POF with one deposition layer (3 layers matrix method), with gold layer ( $20 \mathrm{~nm}$ )

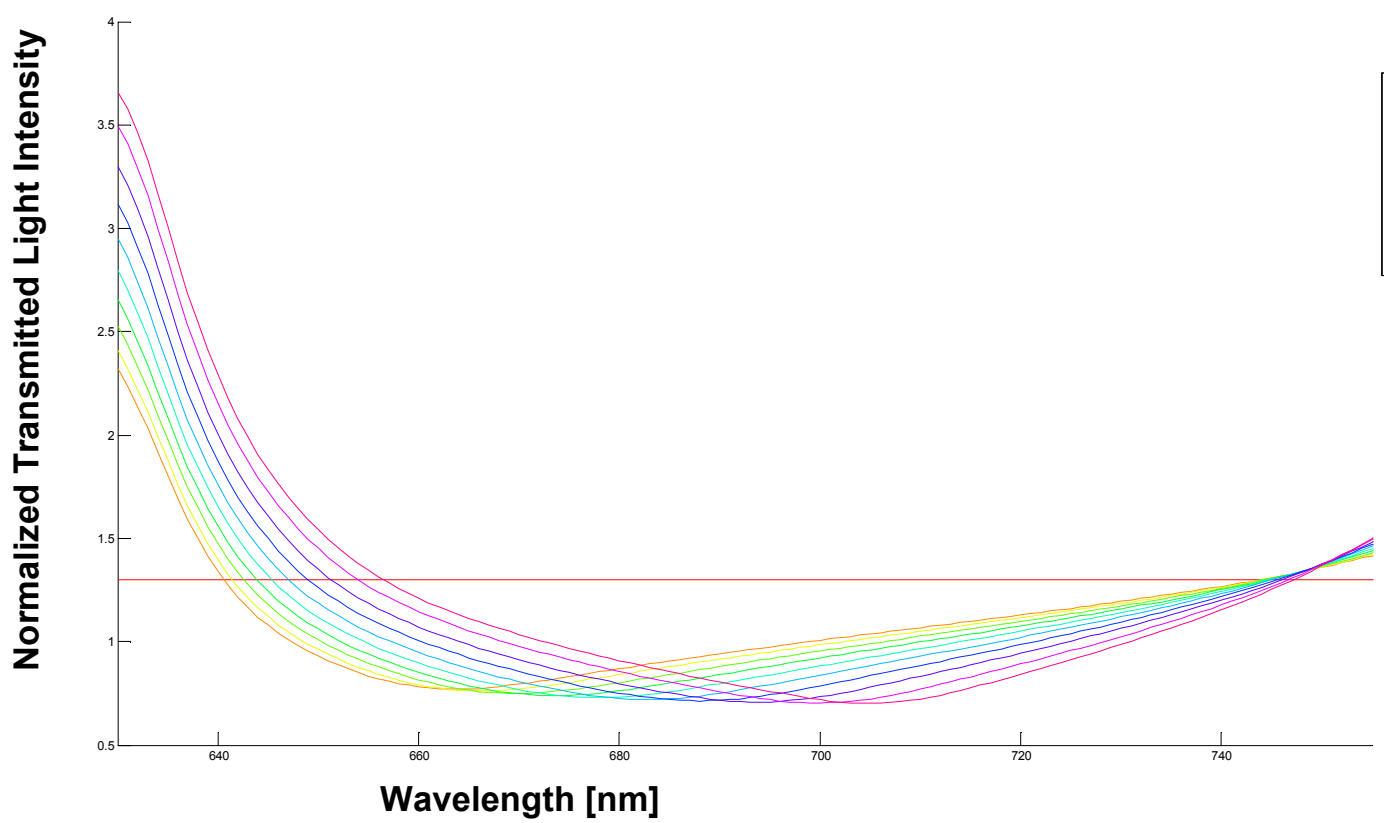

a. spectral response in wavelengths range of interest with different refractive index values (legend)-worst case 


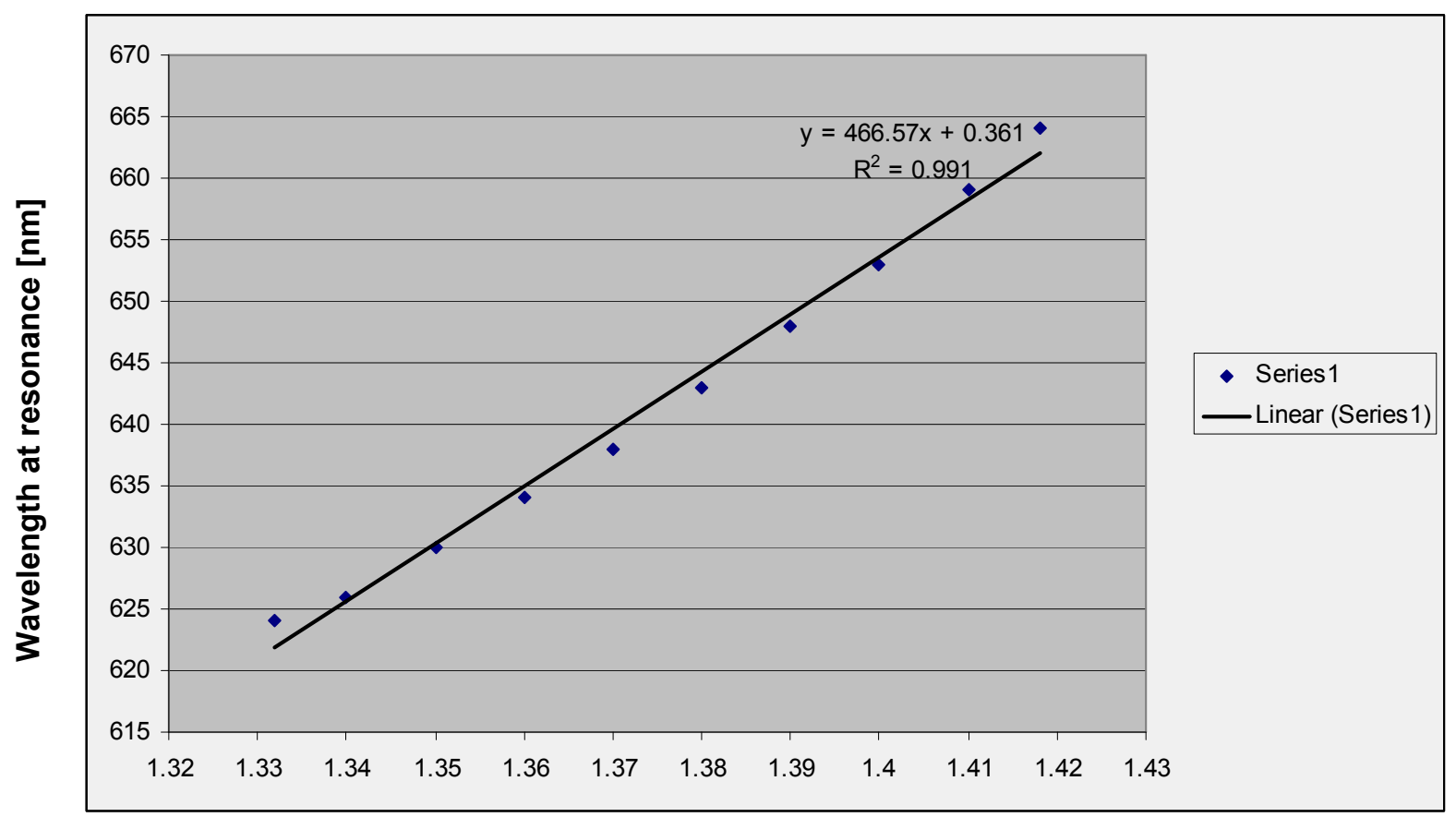

Refractive index [RIU]

b. Linearity of the SPR response

Fig 4. POF with 2 deposition layers(4 layers matrix method), gold layer dimension $\left(\mathrm{d}_{2}\right)-20 \mathrm{~nm}$.

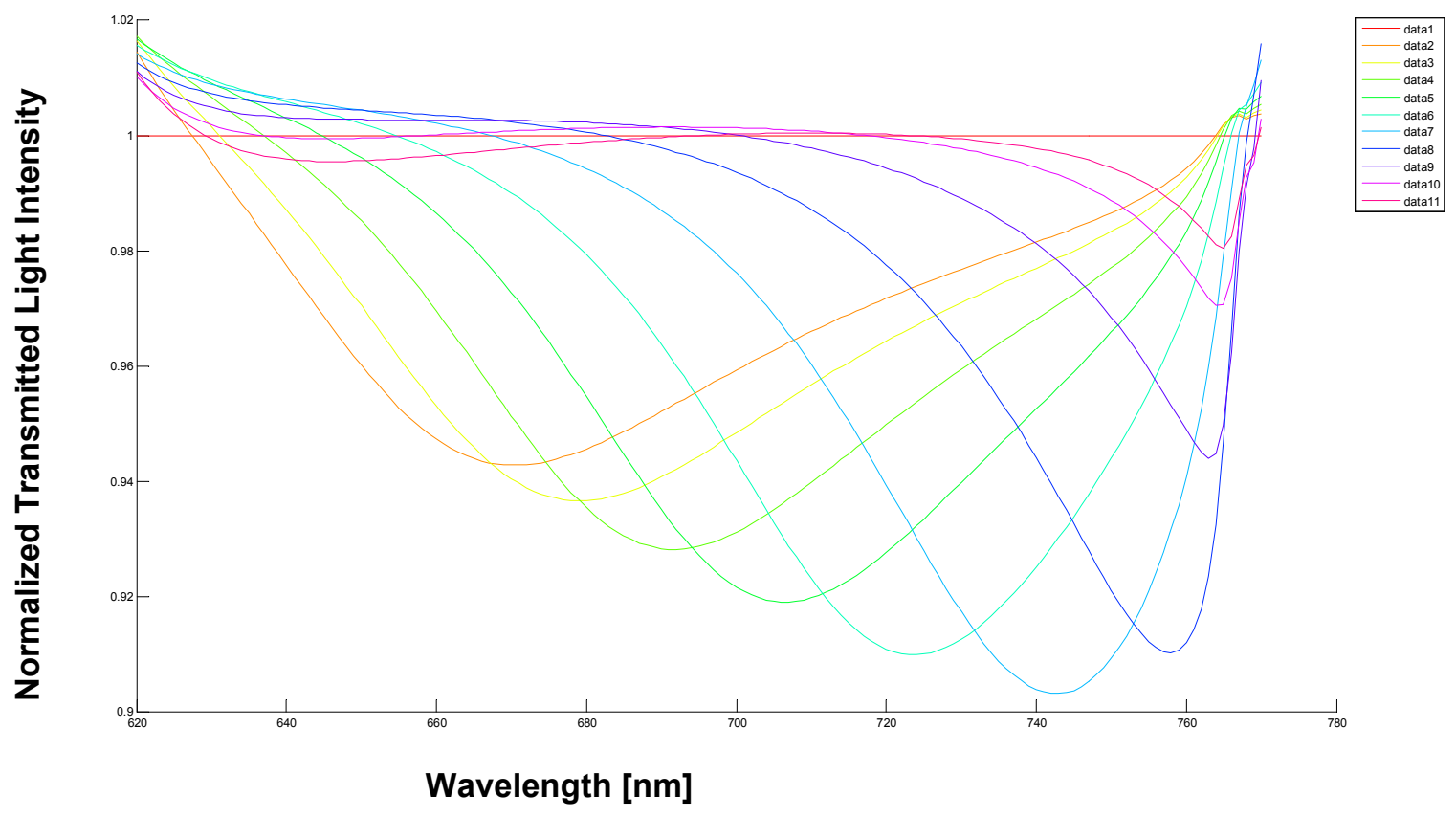

a. spectral response in wavelengths range of interest with different refractive index values (legend) 


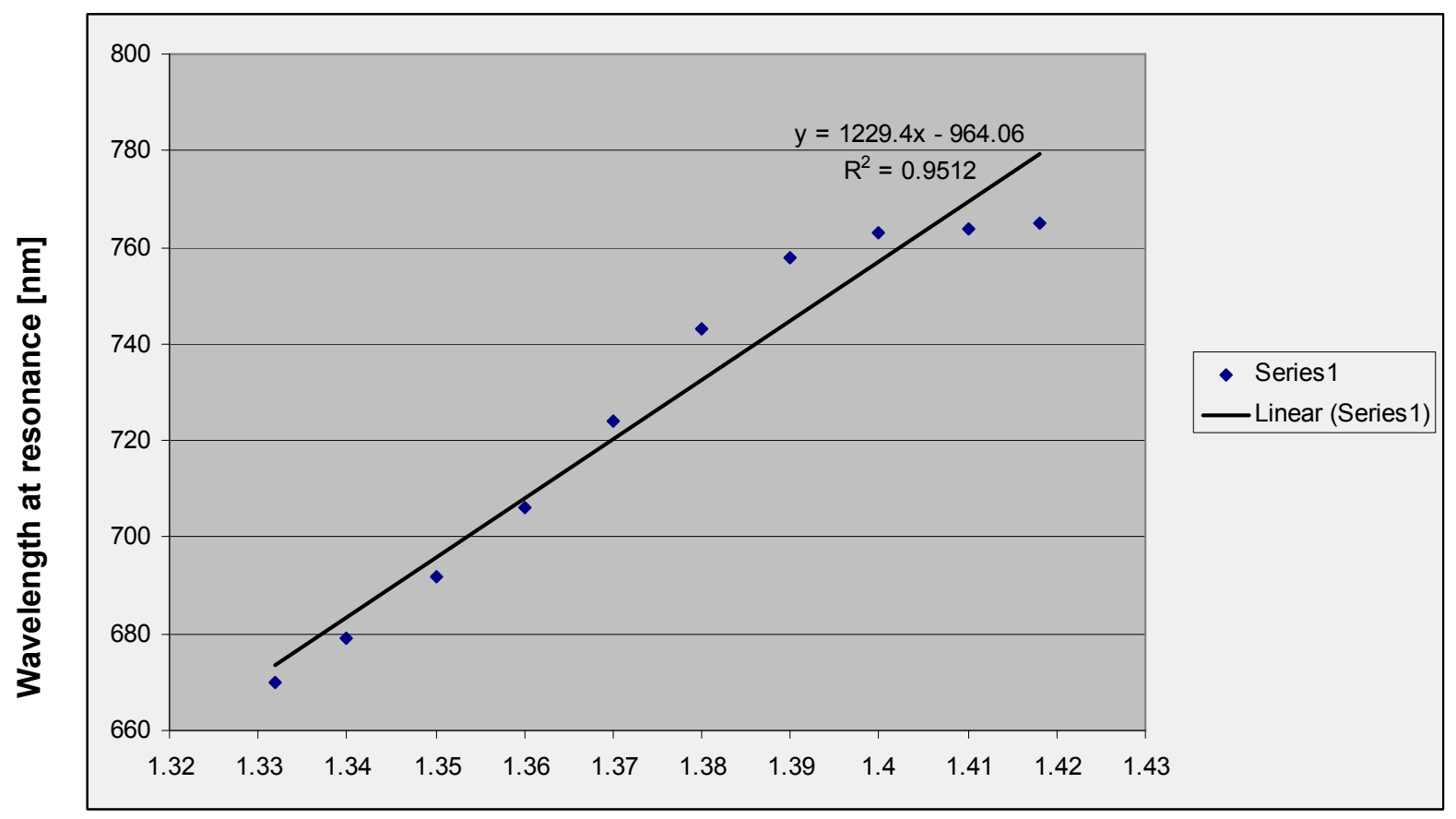

Refractive index [RIU]

b. Linearity of the SPR response

Fig 5. POF with 4 layers stack structure on sensing region, with gold layer dimension $\left(d_{2}\right.$ and $\left.d_{4}\right)-$ with different configuration and buffer layer dimension $\left(\mathrm{d}_{1}\right.$ and $\left.\mathrm{d}_{3}\right)$ in different gold to gold ratio $(60 \mathrm{~nm}$ to $40 \mathrm{~nm})-$ with total stack dimension $1500 \mathrm{~nm}$

Table 1. The performance comparison of different multi-layer geometry of the D-type POF, with refractive index range 1.33-1.418

\begin{tabular}{|c|c|c|c|c|}
\hline Multi-layer type analysis & $\begin{array}{l}\text { Dimensions } \\
{[\mathrm{nm}]}\end{array}$ & $\begin{array}{l}\text { Resolution, } \\
\text { SNR }_{\text {average }}\end{array}$ & $\begin{array}{l}\text { Sensitivity } \\
\text { S } \text { average } \\
\text { [nm/RIU]* } \\
* 10^{\wedge} 3\end{array}$ & $\overline{\mathbf{R}^{2}}$ \\
\hline \multirow[t]{3}{*}{$\begin{array}{l}\text { 3-layers (core/gold/analyt) } \\
\text { Fig. } 3\end{array}$} & $\begin{array}{l}\mathrm{d}_{1}=20 \quad\left(\Delta \mathrm{n}_{\text {sensing }}\right. \\
=0.01)\end{array}$ & $\begin{array}{l}0.04 \\
\left(\mathrm{~d}_{\mathrm{FWHM}}=200 \mathrm{~nm}\right)\end{array}$ & 0.46 & 0.92 \\
\hline & $\mathrm{d}_{1}=40$ & $\begin{array}{l}0.12 \\
\left(\mathrm{~d}_{\mathrm{FWHM}}=150 \mathrm{~nm}\right)\end{array}$ & 2.1 & 0.97 \\
\hline & $\mathrm{d}_{1}=60$ & $\begin{array}{l}0.15 \\
\left(\mathrm{~d}_{\mathrm{FWHM}}=150 \mathrm{~nm}\right)\end{array}$ & 2.4 & 0.97 \\
\hline \multirow{4}{*}{$\begin{array}{l}\text { 4-layers } \\
\text { (core/buffer/gold/analyt) } \\
\text { Fig } 4\end{array}$} & $\begin{array}{l}\mathrm{d}_{2}=20 \\
\mathrm{~d} 1=1.5 \mu \mathrm{m}\end{array}$ & $\begin{array}{l}0.13 \\
\left(\mathrm{~d}_{\mathrm{FWHM}}=65 \mathrm{~nm}\right)\end{array}$ & 0.4 & 0.991 \\
\hline & $\mathrm{d}_{2}=40, \mathrm{~d} 1=1.5 \mu \mathrm{m}$ & $\begin{array}{l}0.08 \\
\left(d_{\mathrm{FWHM}}=100 \mathrm{~nm}\right)\end{array}$ & 0.8 & 0.995 \\
\hline & $\mathrm{d}_{2}=40, \mathrm{~d} 1=3.5 \mu \mathrm{m}$ & $0.1\left(\mathrm{~d}_{\mathrm{FWHM}}=48 \mathrm{~nm}\right)$ & 0.5 & 0.992 \\
\hline & $\begin{array}{l}\mathrm{d}_{2}=60 \\
\mathrm{~d} 1=1.5 \mu \mathrm{m}\end{array}$ & $\begin{array}{l}0.6 \\
\left(d_{\mathrm{FWHM}}=17 \mathrm{~nm}\right)\end{array}$ & 1.1 & 0.98 \\
\hline \multirow[t]{2}{*}{$\begin{array}{l}\text { 5-layer } \\
\text { (core/buffer/silver/gold/analyt) }\end{array}$} & $\begin{array}{l}\mathrm{d}_{3}=40, \\
\mathrm{~d}_{2}=20, \mathrm{~d} 1=1.5 \mu \mathrm{m}\end{array}$ & $\begin{array}{l}0.1 \\
\left(\mathrm{~d}_{\mathrm{FWHM}}=100 \mathrm{~nm}\right)\end{array}$ & 1.2 & 0.98 \\
\hline & $\begin{array}{l}\mathrm{d}_{3}=40, \\
\mathrm{~d}_{2}=40, \mathrm{~d} 1=1.5 \mu \mathrm{m}\end{array}$ & $\begin{array}{l}0.13 \\
\left(\mathrm{~d}_{\mathrm{FWHM}}=85 \mathrm{~nm}\right)\end{array}$ & 1.3 & 0.96 \\
\hline $\begin{array}{l}\text { 6-layer } \\
\text { (core/buffer/silver/buffer/gold/analyt) }\end{array}$ & \multicolumn{4}{|c|}{$\begin{array}{l}\text { The same behavior like 5-layer } \\
\text { (core/buffer/silver/gold/analyt) }\end{array}$} \\
\hline 6-layer & $\mathrm{d}_{4}=40$ & $0.12\left(\mathrm{~d}_{\mathrm{FWHM}}=100\right)$ & 1.1 & 0.98 \\
\hline
\end{tabular}


(core/buffer/gold/buffer/gold/analyt) $\quad \mathrm{d}_{3}=1.5 \mu \mathrm{m}$

Fig 5 .

\begin{tabular}{|c|c|c|c|}
\hline $\begin{array}{l}\mathrm{d}_{4}=40, \\
\mathrm{~d}_{3}=1.5 \mu \mathrm{m} \\
\mathrm{d}_{2}=40, \mathrm{~d} 1=1.5 \mu \mathrm{m}\end{array}$ & $\begin{array}{l}0.179 \\
\left(d_{\mathrm{FWHM}}=80\right)\end{array}$ & 1.2 & 0.96 \\
\hline $\begin{array}{l}\mathrm{d}_{4}=60, \\
\mathrm{~d}_{3}=1.5 \mu \mathrm{m} \\
\mathrm{d}_{2}=20, \mathrm{~d} \mathrm{l}=1.5 \mu \mathrm{m}\end{array}$ & $\begin{array}{l}0.17 \\
\left(\mathrm{~d}_{\mathrm{FWHM}}=80\right)\end{array}$ & 1.2 & 0.96 \\
\hline $\begin{array}{l}\mathrm{d}_{4}=60, \\
\mathrm{~d}_{3}=1.5 \mu \mathrm{m} \\
\mathrm{d}_{2}=40, \mathrm{~d} \mathrm{l}=1.5 \mu \mathrm{m}\end{array}$ & $\begin{array}{l}0.32 \\
\left(\mathrm{~d}_{\mathrm{FWHM}}=40,\right. \\
\text { resolution })\end{array}$ & higher 1.2 & 0.95 \\
\hline
\end{tabular}

\section{Summary}

This paper presents theoretical comparison between different multi-layer geometry of the D-type POF, in order to design the SPR sensor with the best values for sensitivity and resolution of analyt detection. The numerical simulation is based on transfer matrix formalism. It was studied 5 basic structures, to search a geometrically optimized optical fiber-based sensor. In the selected geometry the real capabilities of the manufacture devices are considered. Also, to protect silver layer from oxidation, the gold layer is consider as the outer coating (and structures with silver as the outer coating are neglected).

From theoretical and experimental results, the SPR sensor based on POF demonstrates good linearity and good proportional correlation $\left(\mathrm{R}^{2}\right.$ in $0.92-0.99$ range) with $\mathrm{RI}$ values in range 1.331.40 for the analyt. Because of the good agreement between the tested geometry and theoretical results, numerical simulations could be used to predict better functioning conditions of SPR sensor.

\section{Acknowledgments}

1.a.* The work was supported by the project "Development and support of multidisciplinary postdoctoral programmes in major technical areas of national strategy of Research-DevelopmentInnovation" 4D-POSTDOC, contract no. POSDRU/89/1.5/S/52603, co-funded by the European Social Fund through Sectoral Operational Programme Human Resources Development 2007-2013 and

2.b.** sensor developed is supported by the project PON01_01525 "MONICA".

\section{References}

[1] Nunzio Cennamo, Davide Massarotti, Ramona Galatus, Laura Conte, Luigi Zeni, Performance Comparison of Two Sensors Based on Surface Plasmon Resonance in a Plastic Optical Fiber, Sensors 13 (2013), 721-735.

[2] Charles Kittel, Introduction to Solid State Physics. 8-th edition, Willey\&Sons, USA, 2005, pp.393-424.

[3] S. N. Kasarova et al. Analysis of the dispersion of optical plastic materials, Optical Materials 29, 2007, pp. 1481-1490.

[4] Electronic Materials, Microelectronic Technologies, Information on Figure 3, dispersion curve http://www.microresist.de/products/room_haas/pdf/Microposit_S1800_G2_Serie.pdf

[5] Max Born, E. Wolf, Principles of Optics, Cambridge Press, 7-th edition, reprinted 2002, pp. 5863.

[6] Bora Ung and Yunlong Sheng, Interference of surface waves in a metallic nanoslit, Optics Express, Vol. 15, No. 3, 2007, pp. 1182-1190 
[7] Rakic et al., Optical properties of metallic films for vertical-cavity optoelectronic devices, Applied Optics, Vol. 37, No. 22 , 1998, pp.5271-5283

[8] Navneet K. Sharma, Performances of different metals in optical fibre-based surface plasmon resonance sensor, Springer Pramana Journal of Physics, Vol. 78, No. 3, March 2012, pp. 417-427

[9] Ming-Hung Chiu, Chih-Hsien Shih, Searching for optimal sensitivity of single mode D-type optical fiber sensor in the phase measurement Sensors and Actuators B 131, 2008, pp. 596-601

[10] Mitsuhiro Iga, Atsushi Seki, Kazuhiro Watanabe ${ }^{a}$, Gold thickness dependence of SPR-based hetero-core structured optical fiber sensor, Sensors and Actuators B: Chemical, Volume 106, Issue 1, 29 April 2005, pp. 363-368

[11] Masaru Mitsushio, Kensaku Watanabe, Yasuyuki Abe, Morihide Higo Sensor properties and surface characterization of aluminum-deposited SPR optical fibers, Sensors and Actuators A: Physical, Volume 163, Issue 1, September 2010, pp. 1-8. 